Z. klin. Chem. u. klin. Biochem.

10. Jg. 1972 , S. $86-90$

\title{
Die Zuverlässigkeit der simultanen Natrium-, Kalium- und Chloridbestimmung im Serum mit dem Analysenautomat C 4 (Perkin-Elmer)
}

\author{
Von R. HAECKEL
}

\author{
Institut für Kliniscbe Chemie (Geschäftsfübrender Direktor: Prof. Dr. Dr. J. Büttner) \\ Medizinische Hochscbule Hannover
}

(Eingegangen am 3. September 1971)

Die Zuverlässigkeit der simultanen Natrium-, Kalium- und Chloridbestimmung mit dem Analysenautomat C 4 wurde untersucht. Die von Tag zu Tag Präzisionen von Kontrollseren mit Elektrolytkonzentrationen im Normalbereich lagen zwischen 1,2 und 1,7\%. Urinanalysen eignen sich wegen des engen Meßbereiches der Kaliumbestimmung nicht. Kationische Interferenzen sind bei der Natrium- und Kaliumbestimmung gering und können vernachlässigt werden. Fluoridionen führen in hohen Konzentrationen bei Chlorid zu einem negativen, Jodidionen zu einem positiven Fehler.

\section{The reliability of the simultaneous determination of sodium, potassium and cbloride in serum with the automatic analyser C 4 (Perkin Elmer)}

The reliability of the simultaneous determination of sodium, potassium and chloride in serum with the automatic analyser $\mathrm{C} 4$ was investigated. The day to day precision of control sera with electrolyte concentrations in the normal tange was between 1.2 and $1.7 \%$. Owing to the narrow measurement range for potassium, the method is not suitable for urine. In the determination of sodium and potassium, interference by cations is low and may be ignored. High concentrations of fluoride ions result in a negative error in the determination of chloride and a positive error for iodide.

Der Analysenautomat C 4 (Bodenseewerk Perkin-Elmer $\mathrm{GmbH}$, Überlingen) ist ein Vierkanalgerät, das mit einem Emissionsflammenphotometer ausgerüstet werden kann. Dabei werden die Natrium- und Kaliumkonzentrationen aus einer Verdünnung im ersten Kanal gemessen, so daß noch weitere drei Kanäle für photometrische Methoden zur Verfügung stehen. Wir bestimmen zusätzlich im 2. und 3. Kanal Chlorid mit dem Rhodanidverfahren (1), das von CLAuss und Mitarbeitern. (2) für das C 4-Gerät adaptiert wurde. Im folgenden werden unsere Erfahrungen über die Zuverlässigkeit der Elektrolytbestimmungen im Serum mit diesem Gerät berichtet.

\section{Methodik \\ Einstellung des Gerätes}

Die Bedienung des Gerätes und das Dosierschema entsprechen den Angaben des Herstellers. Das Flammenphotometer verbraucht etwa 0,51 Propan und 91 Luft pro Minute. Als internen Standard verwenden wir eine $\mathrm{Li}_{2} \mathrm{SO}_{4}$-Lösung $(1 \mathrm{mMol} / \mathrm{l})$, die Caesiumchlorid $(20 \mu \mathrm{Mol} / \mathrm{l})$ enthält und mit der die Proben 1:201 verdünnt werden. Beim Rhodanidverfahren wurde lediglich die Konzentration des $\mathrm{HgCl}_{2}$ im Chloridreagenz von 0,75 auf $1,289 \mathrm{mMol} / \mathrm{l}$ erhöht, um den linearen Bereich der Eichkurve zu erweitern. Der 1 . Kanal wird mit $0,1 \mathrm{~N} \mathrm{HCl}$ und anschließend zweimal mit bidest. Wasser, der 2. und 3. Kanal dreimal mit bidest. Wasser gespült.

\section{Eichlösung}

Die Eichung des Gerätes effolgt mit einer einzigen wäßr. Lösung, die $\mathrm{NaCl}$ und $\mathrm{KCl}$ enthält:

$$
\begin{array}{lr}
\text { Natrium } & 140 \mathrm{mMol} / 1 \\
\text { Kalium . } & 5 \mathrm{mMol} / 1 \\
\text { Chlorid } & 145 \mathrm{mMol} / \mathrm{l}
\end{array}
$$

Beide Salze werden etwa $12 \mathrm{Stdn}$. im Vakuum über Phosphorpentoxid getrocknet. Eichlösung und bidest. Wasser zur Ein- stellung des Nullpunktes (Blindlösungen sind nicht erforderlich) werden wie Proben behandelt.

Beschickung der Probengefäßsegmente

\begin{tabular}{crl}
\hline $\begin{array}{c}\text { Fortlaufende } \\
\text { Nummer }\end{array}$ & & \multicolumn{1}{c}{ Proben } \\
\hline $1-8$ & $8 \times$ & Bidest. Wasser \\
$9-20$ & $12 \times$ & Standardlösung \\
$21-23$ & $3 \times$ & Kontrollserum (Präzisionskontrolle) \\
$24-44$ & $21 \times$ & Proben (einschl. Richtigkeitskontrolle) \\
$45-47$ & $3 \times$ & Standardlösung (Driftkontrolle) \\
$48-52$ & $5 \times$ & $\begin{array}{l}\text { Bidest. Wasser (evtl. Korrektur des Null- } \\
\text { punktes) }\end{array}$ \\
$53-60$ & $8 \times$ & $\begin{array}{l}\text { Standardlösung (Uberprüfung der } \\
\end{array}$ \\
$61-84$ & $24 \times$ & $\begin{array}{l}\text { Eichung) } \\
\text { Proben } \\
85-87\end{array}$ \\
$3 \times$ & Standardlösung (Driftkontrolle)
\end{tabular}

ab Nr. 88 werden die Proben wie zwischen Nr. 48 und 87 angeordnet. Probenfrequenz: 45 Proben/Std.

\section{Materialien}

Rinderalbumin (reinst) wurde von den Behringwerken (Marburg), die Kontrollseren Hyland normal und abnormal von Travenol International GmbH (München), Seronorm von Dr. Molter GmbH (Heidelberg), Monitrol, Pathotrol und Labtrol von Merz und Dade (München), Versatol von Goedecke AG (Freiburg), SMA-12 Referenzserum von der Firma Technicon (Frankfurt), alle anderen Chemikalien wurden von der Firma E. Merck AG (Darmstadt) bezogen.

\section{Ergebnisse}

\section{Meßbereich}

Bei der Natriumbestimmung verläuft die Eichkurve in dem im Serum vorkommenden Bereich von 110 bis $160 \mathrm{mMol} / 1$ linear (Abb. 1a). Die Linearität erstreckt sich jedoch nicht exakt auch auf den tieferen Konzentrationsbereich. Umgekehrt biegt die Eichkurve bei 

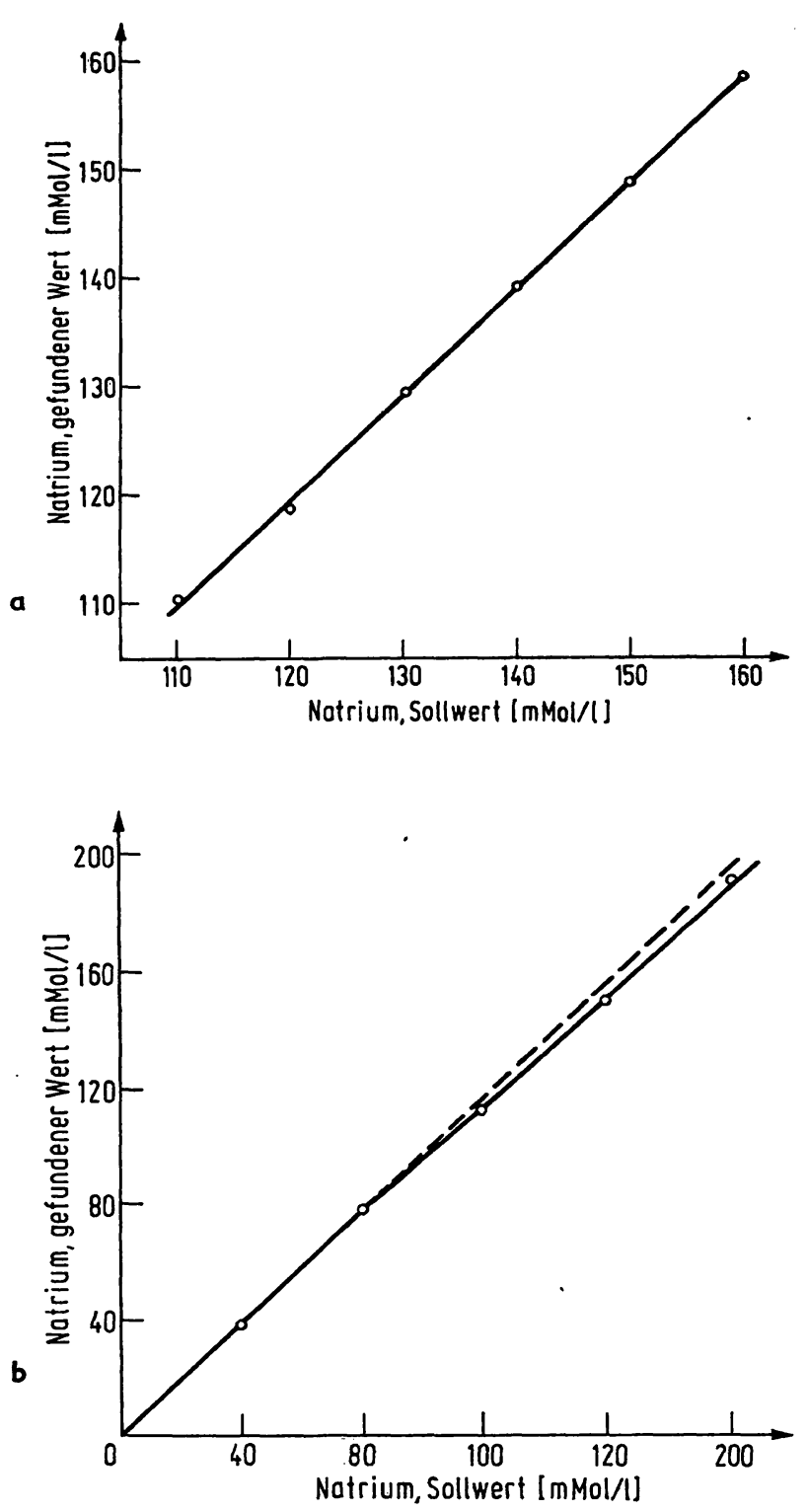

Abb. 1

Eichkurve der Natriumbestimmung. Einstellung des Gerätes bei a) mit der üblichen Standardlösung, bei b) mit einer kaliumfreien Natriumlösung ( $40 \mathrm{mMol} / \mathrm{l})$

$80 \mathrm{mMol} / \mathrm{l}$ leicht ab, wenn die Anzeige des Flammenphotometers mit einer Lösung von $40 \mathrm{mMol} / 1$ Natrium eingestellt wird ( $\mathrm{Abb}$. $1 \mathrm{~b}$ ).

Die Eichgerade für Kalium verläuft nicht durch den Nullpunkt, wenn die Anzeige des Flammenphotometers mit einem wäßrigen Standard, der nur $5 \mathrm{mMol} / 1$ $\mathrm{KCl}$ enthält, eingestellt wird. Konzentrationen über $5 \mathrm{mMol} / 1$ werden zu hoch, tiefere Konzentrationen zu niedrig angezeigt (Abb. 2). Da ein Abbiegen der Eichkurve nach oben durch Ionisationseffekte (4) bewirkt werden kann, setzten wir den Standardlösungen Caesiumchlorid zu, das die Natrium- und Kaliumbestimmung unter unseren experimentellen Bedingungen nicht wesentlich stört. Eine Konzentration von $40 \mathrm{mMol} / 1$, die 10 fach höher als die von uns verwendete liegt, bewirkt bei Natrium keine sichtbare Anzeige, bei Kalium einen Ausschlag, der 0,1 mMol/1 entspricht. In Anwesenheit von Caesiumchlorid verläuft die Kalium-Eichkurve etwas flacher, und die Sollwerte werden zwischen 3 und

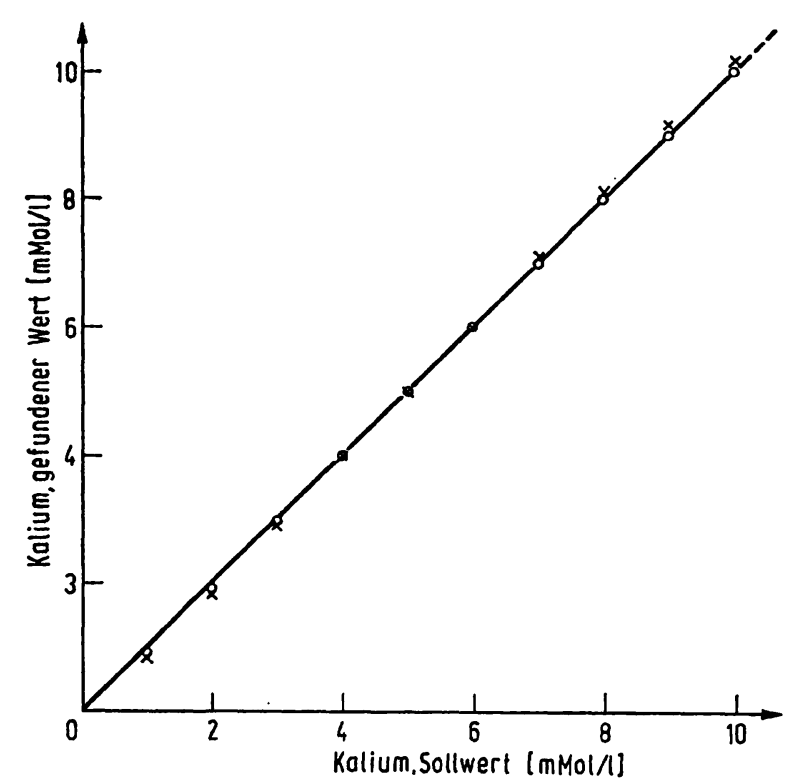

Abb. 2

Eichkurve der Kaliumbestimmung. Die Einstellung des Flammenphotometers erfolgte mit bidest. $\mathrm{H}_{2} \mathrm{O}$ und einem wäßr. Standard (5 mMol/1). Kreise: Mittelwerte aus 7 Bestimmungen an verschiedenen Tagen; die Verdünnungslösung wurde mit Caesiumchlorid $(20 \mu \mathrm{Mol} / 1)$ versetżt. Kreuze: die Verdünnungslösung enthielt kein $\mathrm{CsCl}_{2}, \mathrm{n}=4$

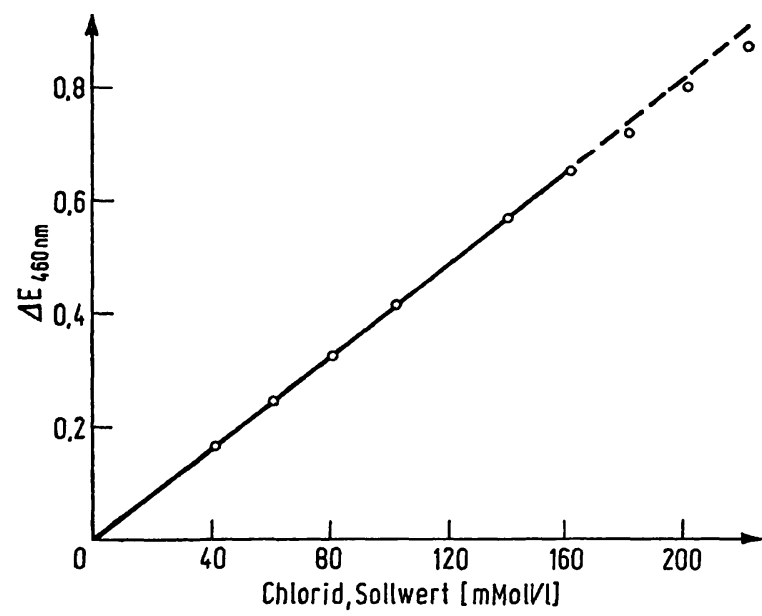

Abb. 3

Eichkurve der Chloridbestimmung, gemessen bei $460 \mathrm{~nm}$

$10 \mathrm{mMol} / 1$ weitgehend erreicht (Abb. 2). Lediglich bei tieferen Konzentrationen liegt die Anzeige ebenfalls zu niedrig (bei $2 \mathrm{mMol} / 1$ um 3\%). Auf Grund dieses Versuches setzen wir der Lithium-haltigen Verdünnungslösung Caesiumchlorid zu.

Die Eichkurve bei der Chloridbestimmung verläuft bei unserer Modifikation bis $160 \mathrm{mMol} / \mathrm{l}$ so gut wie linear (Abb. 3).

Die Nachweisgrenzen wurden als die 3 fache Standardabweichung von Wasserwerten (3) in der Serie bestimmt und betrugen für Chlorid $1,2 \mathrm{mMol} / 1(\mathrm{n}=20$ ), für Natrium 0,4 mMol/1 $(n=20)$ und für Kalium 0,05 mMol/1 $(\mathrm{n}=20)$.

\section{Präzision}

Standardlösungen und Kontrollseren wurden täglich 3 fach analysiert. Aus den 2. und 3. Werten wurde die Präzision in der Serie und von Tag zu Tag errechnet (Tab. 1). 
Tab. 1

Präzision der Natrium-, Kalium- und Chloridbestimmung am Analysenautomat C 4

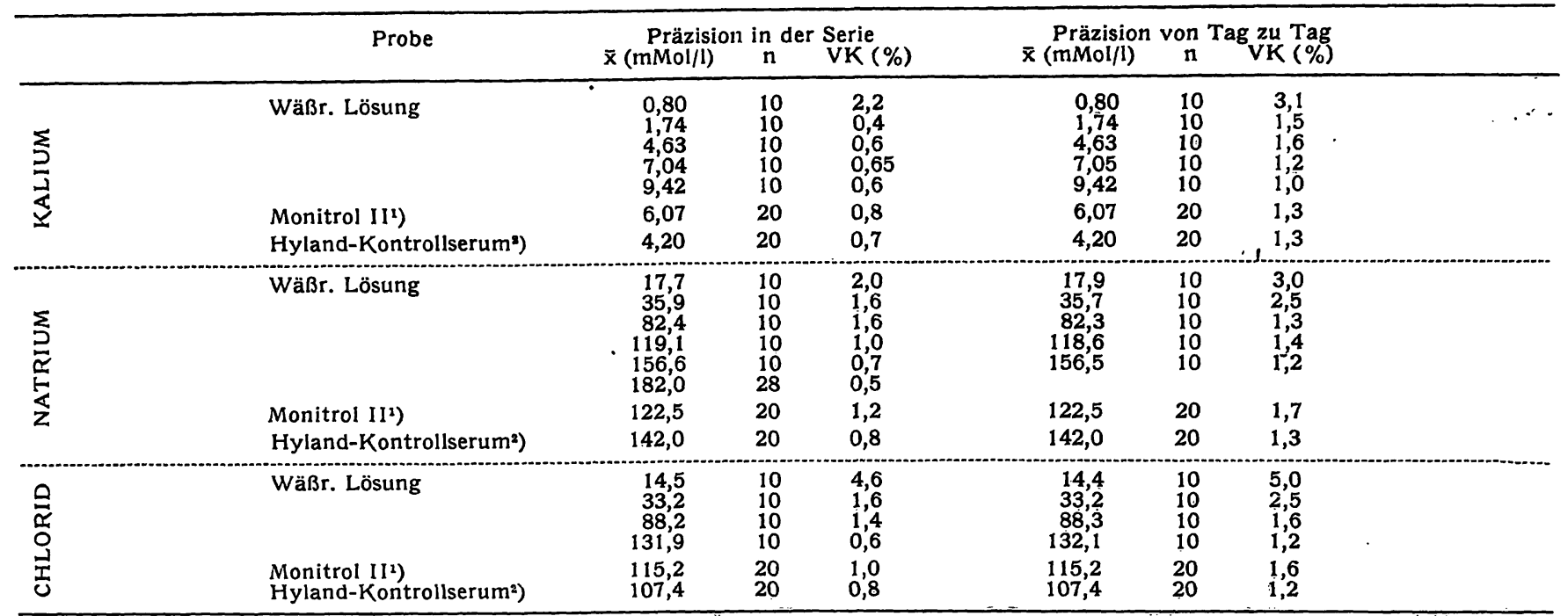

2) Chargen-Nr. PTD $24 \mathrm{C} . \quad$ 2) Chargen-Nr. 8 A 1.

\section{Richtigkeit}

Die Richtigkeit der Elektrolytbestimmungen wurde mit 9 verschiedenen Kontrollseren überprüft (Abb. $4 a-c)$.

\section{Interferenzen}

Kationische Interferenzen sind bei der Flammenphotometrie schon lange bekannt (4). Ein signifikanter Einfluß von Kaliumionen auf die Natriumbestimmung (Tab. 2) wurde am C 4-Gerät nicht festgestellt.

Umgekehrt verfälschen Natriumionen die Kaliumbestimmung in stärkerem Maße (Tab. 3). $\mathrm{Da}$ die Natriumionen im Humanserum jedoch nur um maximal $50 \mathrm{mMol} / \mathrm{l}$ schwanken, beträgt der absolute Fehler bei einer mittleren Kaliumkonzentration von $5 \mathrm{mMol} / 1$ maximal $\pm 1 \%$ und kann daher vernachlässigt werden.

$\mathrm{Da}$ Lithiumionen bei der Flammenphotometrie als interner Standard dienen, sollten die Proben für die Natrium- und Kaliumbestimmung frei von Lithium sein.

Tab. 2

Der Einfluß von Kaliumionen auf die Bestimmung der Natriumkonzentration

\begin{tabular}{ccc}
\hline $\begin{array}{c}\text { Sollwert Natrium } \\
\text { (mMol/l) }\end{array}$ & $\begin{array}{c}\text { Kalium- } \\
\text { konzentration } \\
\text { (mMol/l) }\end{array}$ & $\begin{array}{c}\text { Gefundene } \\
\text { Natriumkonzentration } \\
\overline{\mathbf{x}} \pm \mathbf{S}(\text { mMol/l) } \\
(\mathrm{n}=4)\end{array}$ \\
\hline 130 & 0 & $129,5 \pm 1,3$ \\
130 & 5 & $129,5 \pm 1,0$ \\
130 & 100 & $131,0 \pm 0,8$ \\
130 & 200 & $131,2 \pm 1,5$ \\
\hline
\end{tabular}

Tab. 3

Der Einfluß von Natriumionen auf die Kaliumbestimmung

\begin{tabular}{ccc}
\hline $\begin{array}{c}\text { Sollwert Kalium } \\
(\mathrm{mMol} / 1)\end{array}$ & $\begin{array}{c}\text { Natrium- } \\
\text { konzentration } \\
(\mathrm{mMOl} / \mathrm{l})\end{array}$ & $\begin{array}{c}\text { Gefundene } \\
\text { Kaliumkonzentration } \\
\overline{\mathbf{x}} \pm \mathbf{s}(\mathrm{mMol} / \mathrm{l}) \\
(\mathrm{n}=4)\end{array}$ \\
\hline 5 & 0 & $5,04 \pm 0,07$ \\
5 & 40 & $5,16 \pm 0,03$ \\
5 & 80 & $5,20 \pm 0,03$ \\
5 & 120 & $5,29 \pm 0,05$ \\
5 & 160 & $5,33 \pm 0,02$ \\
5 & 200 & $5,38 \pm 0,04$ \\
\hline
\end{tabular}

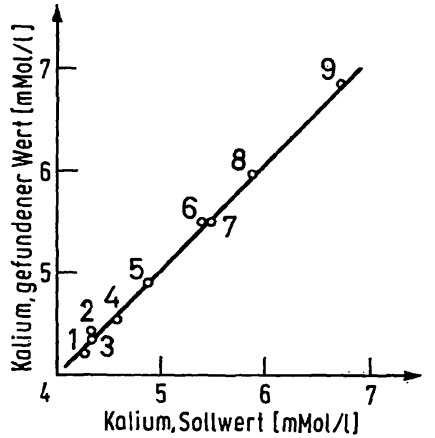

$\mathrm{Abb}, 4 \mathrm{a}$

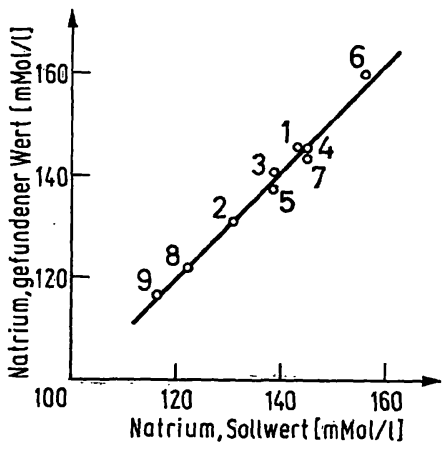

Abb. 4 b

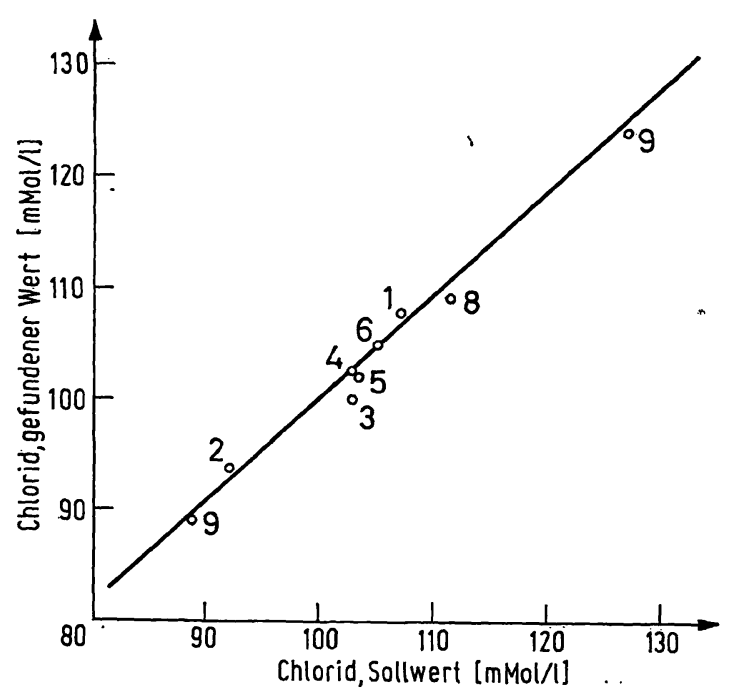

Abb. 4c

Abb. 4

Richtigkeitsprüfung der Kalium- (a), Natrium- (b) und Chloridbestimmụng (c) mit verschiedenen käuflichen Kontroilseren 1 Hyland normal (Chargen Nr. $20 \mathrm{A1}$ ), 2 Seronorm (Nr. 112), 3 Monitrol I (Nr. $103 \mathrm{C}), 4$ Labtrol (Nr. LTD $35 \mathrm{~A}$ ), 5 Versatol (Nr. 2774069 ) yland normal (Nr. 14 Al), 7 Technicon Referenzserum (Nr. BOC 032), 8 Monitrol II (Nr. $23 \mathrm{C}$ ), 9 Pathotrol (Nr. $64 \mathrm{Z}$ ) Regressionsgeraden und Korrelationskoeffizienten:

$\mathrm{K}: \mathrm{y}=1,056 \mathrm{x}-0,2574 ; \quad \mathrm{r}=0,9965$ $\begin{array}{rlr}\text { Na: } y=1,043 x-5,295 ; & r=0,9905 \\ \text { Cl: } y=0,8969 x+9,7878 ; & r=0,9896\end{array}$

Z. klin. Chem. u. klin. Biochem. / 10. Jahrg. 1972/ Heft 2 
Tab. 4

Der Einfluß von Lithiumionen auf die Natrium- und Kaliumbestimmung

\begin{tabular}{ccc}
\hline $\begin{array}{c}\text { Lithium } \\
(\mathrm{mMol} / \mathrm{l})\end{array}$ & $\begin{array}{c}\text { Gefundene } \\
\text { Natriumkonzen- } \\
\text { tration } \\
(\mathrm{mMol} / \mathrm{l})\end{array}$ & $\begin{array}{c}\text { Gefundene } \\
\text { Kaliumkonzentration } \\
(\mathrm{mMol} / \mathrm{l})\end{array}$ \\
$\overline{\mathrm{x}} \pm \mathrm{s}(\mathrm{n}=4)$ & $\mathrm{x} \pm \mathrm{n}=5)$ \\
\hline 0 & $127,0 \pm 1,0$ & $4,55 \pm 0,05$ \\
5 & $126,5 \pm 1,0$ & $4,48 \pm 0,04$ \\
10 & $123,8 \pm 0,9$ & $4,43 \pm 0,03$ \\
\hline
\end{tabular}

Eine Konzentration von $2 \mathrm{mMol} / \mathrm{l}$ Lithium führt bei Kalium zu einem negativen Fehler von etwa $2,5 \%$, bei Natrium von etwa $2 \%$ (Tab. 4). Normalerweise liegt die Lithiumkonzentration im Serum zwischen 0,4 und $6,3 \mu \mathrm{Mol} / 1$ (5). Nach PyBus und Bowers (6) sollte bei der Behandlung von manischen Patienten eine Konzentration von 1 bis $1,5 \mathrm{mMol} / 1$ erzielt werden. Nur bei Überdosierung werden Serumwerte bis $4 \mathrm{mMol} / \mathrm{l}$ (6) erreicht, die die Natrium- und Kaliumwerte dann geringgradig verfälschen. Die Chloridbestimmung wird durch Lithiumionen nicht beeinflußt.

Bei der Chloridbestimmung ist die Interferenz durch andere Halogene hinlänglich bekannt und mehrfach untersucht worden $(2,7)$. Bromid- und Jodidionen führen zu einem positiven, Natriumfluorid (in hohen Konzentrationen) zu einem negativen Fehler (Tab. 5).

Tab. 5

Der Einfluß von Natriumjodid, Natriumfluorid, Cholesterin und Bilirubin auf die Chloridbestimmung. Alle Resultate wurden mit eine amperometrischen Titrationsmethode ${ }^{1}$ ) verglichen. Es handelt sich um Mittelwerte (mMol/l) mit Standardabweichung aus 5 Bestimmun gen an verschiedenen Tagen

\begin{tabular}{lcc}
\hline \multicolumn{1}{c}{ eingesetzt } & $\begin{array}{c}\mathrm{C} 4 \\
\text { gefunden }\end{array}$ & $\begin{array}{c}\text { Titrator } \\
\text { gefunden }\end{array}$ \\
\hline Chlorid $100 \mathrm{mMol} / 1$ & $100,8 \pm 0,2$ & $\left.99,0^{2}\right)$ \\
Jadid $100 \mathrm{mMol} / 1$ & $88,4 \pm 1,6$ & $\left.98,0^{2}\right)$ \\
$\begin{array}{l}\text { Clorid } 100 \mathrm{mMol} / 1 \\
\quad+\text { Fluorid } 100 \mathrm{mMol} / 1\end{array}$ & $86,0 \pm 0,5$ & $\left.98,7^{2}\right)$ \\
$\begin{array}{l}\text { Chlorid } 100 \mathrm{mMol} / 1 \\
\left.\quad+\text { Cholesterin 10,3 } \mathrm{mMol} / \mathrm{l}^{3}\right)\end{array}$ & $109,4 \pm 1,6$ & $108,8 \pm 1,2$ \\
$\begin{array}{l}\text { Chlorid } 83 \mathrm{mMol} / 1 \\
\left.\quad+\text { Bilirubin } 170 \mu \mathrm{Mol} / \mathrm{1}^{4}\right)\end{array}$ & $85,1 \pm 1,1$ & $84,4 \pm 0,9$ \\
\hline
\end{tabular}

2) Printout clinical chloride titrator (American Instruments Co., Silver Spring, USA).

2) mMol/1, Mittelwert aus Doppelbestimmungen an 2 Tagen

) Cilirubin control Serum, Merz und Dade (Chargen Nr. BIC 916).

Nach Hamilton (7) kann auch Cholesterin das Rhodanidverfahren beeinflussen. Unter unseren Bedingungen trat dieser Effekt bei einer Cholesterinkonzentration, die im Normalbereich für menschliches Serum liegt, nicht auf (Tab. 5). Bilirubin stört die Chloridbestimmung ebenfalls nicht.

\section{Drift}

Auf Grund von Vorversuchen über die Gerätedrift in der Serie beschicken wir die Segmente wie unter Methodik angegeben und führen eine tägliche Driftkontrolle durch, deren Daten wie von BürTNER vorgeschlagen (8) in eine Range-Kontrollkarte eingetragen werden ( $\mathrm{Abb}$. 5). Bei Chlorid und Kalium betrug die Drift nach 32 Proben im Durchschnitt $(n=25)$ etwa $1 \%$, bei Natrium lag sie unter $1 \%$.

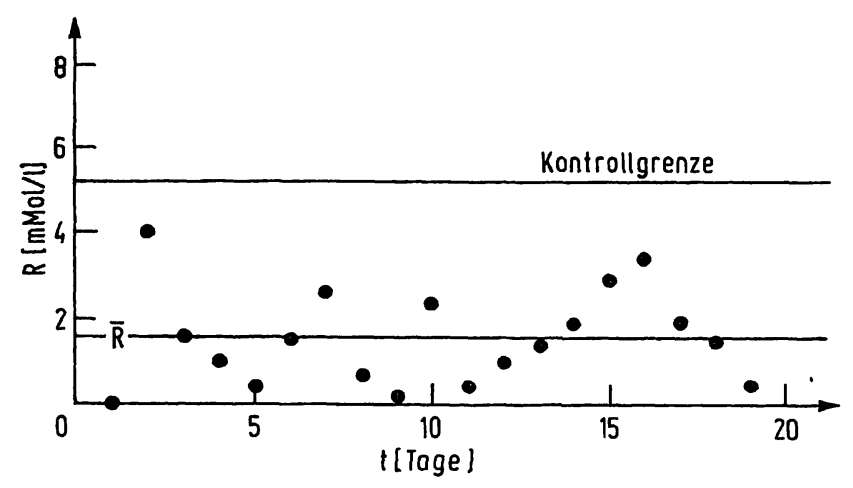

Abb. 5

Range Kontrollkarte zur Überwachung der Gerätedrift von Tag zu Tag. Der einzelne R-Wert. wurde aus der Differenz

$$
\frac{c_{40}+c_{47}}{2}-\frac{c_{10}+c_{20}}{2} \text {, }
$$

die Kontrollgrenze aus $\bar{R} \cdot 3,27$ (11) berechnet

\section{Verschleppung}

Bei dem Analysenautomat C 4 kann es sowohl durch die Probeaufnahme als auch durch die Überführung der aufbereiteten Proben in das Photometer zu Verschleppungseffekten kommen.

Nach experimenteller Ermittlung des Verschleppungskoeffizienten und der methodischen Streuung kann der Konzentrationsbereich abgeschätzt werden, in dem Verschleppungseffekte vernachlässigt werden können. Über diese Befunde wird in der folgenden Mitteilung (9) berichtet.

Neben der Verschleppung von Probe zu Probe kann es theoretisch auch zu einer zyklischen Verschleppung kommen, wenn die Reaktionsgefäße nicht austeichend gespült werden. Dadurch würden die Ergebnisse jeweils nach einem 60-Probenzyklus verfälscht werden. Um dies zu überprüfen, beschickten wir das Gerät nach 10 Poolseren mit 65 Wasserproben. Dabei wurde kein Anstieg der 50. bis 60. Wasserwerte sichtbar, auch dann nicht, wenn die Reaktionsgefäße nur einmal gespült wurden.

Elektrolytbestimmung im Urin

Während Natrium und Chlorid im unverdünnten Urin bestimmt werden können, ist für Kalium meist eine Verdünnung von 1:15 erforderlich. Dabei muß allerdings oft noch eine 2 . Verdünnung (1:10 oder $1: 20)$ vorgenommen werden, da die Kaliumwerte im Urin zwischen 5 und $200 \mathrm{mMol} / \mathrm{l}$ schwanken (10).

Zur Bestimmung der Natrium- und Chloridkonzentration im Utin kann die gleiche Eichlösung wie bei Serumproben verwendet werden. Für Kalium sollte ein Standard gewählt werden, dessen Natriumkonzentration niedriger (z. B. $15 \mathrm{mMol} / \mathrm{l}$ ) liegt als bei Serumanalysen. $20 \mathrm{mMol} / 1$ Natrium bewirken in einem $5 \mathrm{mMol} / \mathrm{l}$ Kaliumstandard einen positiven Fehler von etwa $1 \%$. Unter diesen Bedingungen wurde bei der Natrium- und Chloridbestimmung die gleiche von Tag zu Tag Präzision in gepooltem Patientenurin und in gefriergetrocknetem Kontrollurin erreicht wie bei Serumanalysen. Die Kaliumwerte streuten infolge des zusätzlichen Verdünnungsschrittes etwas stärker. 


\section{Diskussion}

Das Flammenphotometer des Analysenautomaten C 4 ist sehr einfach zu bedienen, wenig störanfällig und liefert zuverlässige Ergebnisse, die in Konzentrationseinheiten ausgedruckt werden. Der enge Bereich der Kaliumanzeige macht das Gerät allerdings für die Elektrolytbestimmung im Urin ungeeignet. Durch die erforderlichen manuellen Verdünnungsschritte werden die Vorteile der Mechanisierung aufgehoben. Bei Serumanalysen umfaßt der lineare Meßbereich des Gerätes die in-vivo vorkommende Konzentrationen. Lediglich bei pathologisch niedrigen Kaliumkonzentrationen ist mit einem geringen negativen Fehler zu rechnen.

\section{Literatur}

1. ZALL, M. D., D. Fischer und M. D. GARner, Analytic. Chem. 28, 1665 (1956). - 2. Clauss, J., E. Graf, J. Ringhardtz und A. Schmitr, Berichte zur Klinischen Chemie, Heft 5 (1969), Hrsg.: Bodenseewerk Perkin-Elmer GmbH, Überlingen. - 3. KaIser, H., Z. analyt. Chem. 209, 1 (1965). - 4. HerrmanN, R. und C. Th. Alkemade, Flammenphotometrie, 2. Aufl. Springer, Berlin-Göttingen-Heidelberg (1960). - 5. GEIGY-Tabellen, 7.
Auflage, 1968, p. 563. - 6. Pybus, J. und G. N. Bowers, Clin. Chem., New York 16, 139 (1970). - 7. Hamriton, R. H., Clin. Chem. New York 12, 1 (1966). - 8. BürTNER, H., diese Z. 5 , 41 (1967). - 9. Haeckel, R. und A. J. Porth, diese Z. 10, 91 (1972). - 10. Pirke, K. M. und D. Stamm, diese Z. 8, 241 (1970). 11. Grant, E. L., Statistical quality control, MCGRAw-HILL Book Comp., New York, 562 (1964).
Dr. R. Haeckel 3000 Hannover Roderbruchstr. 101 